\title{
A Recursive Legendre Polynomial Analytical Integral Method for the Fast and Efficient Modelling Guided Wave Propagation in Rectangular Section Bars of Orthotropic Materials
}

\author{
Xiaoming Zhang and Shuangshuang Shao \\ School of Mechanical and Power Engineering, Henan Polytechnic University, Jiaozuo 454003, China.
}

\begin{abstract}
Shuijun Shao
School of Mathematics and Information Science, Henan Polytechnic University, Jiaozuo 454003, China. E-mail: shuijunshao@163.com
\end{abstract}

(Received 27 January 2021; accepted 10 June 2021)

\begin{abstract}
Ultrasonic guided waves are widely used in non-destructive testing (NDT), and complete guided wave dispersion, including propagating and evanescent modes in a given waveguide, is essential for NDT. Compared with an infinite plate, the finite lateral width of a rectangular bar introduces a greater density of modes, and the dispersion solutions become more complicated. In this study, a recursive Legendre polynomial analytical integral (RLPAI) method is presented to calculate the dispersion behaviours of guided waves in rectangular bars of orthotropic materials. The existing polynomial method involves a large number of numerical integration steps, and it is often computationally costly to compute these integrals. The presented RLPAI method uses analytical integration instead of numerical integration, thus leading to a significant improvement in the computational speed. The results are compared with those published previously to validate our method, and the computational efficiency is discussed. The full threedimensional dispersion curves are plotted. The dispersion characteristics of propagating and evanescent waves are investigated in various rectangular bars. The influences of different width-to-thickness ratios on the dispersion curves of four types of low-order modes for a rectangular bar of an orthotropic composite are illustrated.
\end{abstract}

\section{INTRODUCTION}

The use of ultrasonic guided waves has been successfully demonstrated in a wide range of nondestructive testing applications. Dispersion curves of guided waves propagating in a given structure are essential for inspection purposes and to obtain information about the material properties. Accurate algorithms for calculating these curves are indispensable tools for engineers engaging in nondestructive assessment and for scientists studying wave phenomena.

The general frequency equations were first derived by Pochhammer and Chree for a cylinder and by Rayleigh and Lamb for an infinite plate with traction-free faces. ${ }^{1-4}$ The dispersion characteristics of infinite plate and cylindrical waveguides have been treated analytically, numerically, and experimentally by several generations of scholars. Many analytical and numerical methods have been developed and successfully deployed to compute guided wave dispersion curves for platelike and cylindrical structures, such as the semi-analytical finite element method, spectral method, stiffness transfer matrix method, reverberation-ray matrix method, and power series technique..$^{5-9}$ An intrinsic feature of such waveguides is the simplicity of the boundaries, as their boundaries consist of one or two similar faces. They have infinite planar or axial dimensions and have a finite dimension in only one direction.

Unlike simple structures, such as plates and (hollow) cylin- ders, a bar with a rectangular cross section has finite dimensions in two directions, i.e., a finite thickness and a finite width. The formation of guided waves in a rectangular bar becomes more complicated because of the intersections of more boundary faces. Thus, it is more difficult to derive and analyze the dispersion relation of a rectangular bar. The first studies on the dispersion of guided waves in rectangular bars were performed by Morse and Booker, which were confined to low frequencies and to a limited ratio of the side lengths. ${ }^{10-13}$ Using the Rayleigh-Ritz method, Nigro and Aalami gave approximate solutions and presented dispersion curves at low frequencies for square and rectangular bars. ${ }^{14,15}$ With the collocation method, Fraser obtained the dispersion curves of a limited range of wavenumbers for a rectangular bar. Using the finite element method, Taweel et al. obtained the dispersive properties of guided waves in bars. ${ }^{16}$ Gunawan and Hirose developed a boundary element method to calculate the dispersion relations of guided waves in bars with arbitrary cross sections. ${ }^{17}$ Using the semi-analytical finite element method, Veres and Sayir determined the dispersion curves of a rectangular wooden bar and related the dispersion curves and material properties. ${ }^{18}$ Hayashi et al. derived the theoretical dispersion curves and wave structures for a bar with an arbitrary cross section. ${ }^{19}$ Manzanares et al. presented a plane wave expansion method to predict the vibrational elastic modes in bars 
with square and circular cross sections. ${ }^{20}$ Using an analytical superposition method, Krushynska and Meleshko investigated guided waves in a rectangular elastic bar. ${ }^{21}$ Most of these previous studies on guided wave behaviours of rectangular bars concentrated almost exclusively on propagating waves, with a limited number of studies devoted to evanescent waves.

Propagating modes are relevant for wave propagation and vibration. These modes have been discussed extensively for various waveguides. Evanescent modes with complex or purely imaginary wavenumbers are also present in perfectly elastic materials without any energy leakage and represent local modes. ${ }^{22}$ The complex spectra of guided waves in simple structures, such as infinite plates and cylinders, have been determined by many researchers using various methods, such as the numerical spectral method, spectral collocation method, semi-analytical method, boundary element method, and Legendre polynomial method. ${ }^{23-28}$ However, very few theoretical studies on evanescent waves in a rectangular bar have been established specifically. Indeed, it is more difficult to obtain dispersion curves for a rectangular bar than for simple plate structures. The recent wide utilization of guided waves in rectangular bars for non-destructive evaluation demands accurate calculations of the complete guided wave dispersion. The traditional methods, e.g., finite element method or root-finding routines, require a far greater coding effort or a tedious iterative search procedure to find complex solutions of the transcendental dispersion equation of guided waves in a rectangular bar. Moreover, although the Legendre polynomial method is quite versatile for guided wave problems, it involves a large amount of numerical integration calculations, resulting in a low computational efficiency. ${ }^{29-34}$

This paper presents a recursive Legendre polynomial analytical integral (RLPAI) method to determine complete roots of the dispersion equation of guided waves in a rectangular orthotropic composite bar. The RLPAI method does not require the large amount of numerical integration calculations required by traditional polynomial methods, and the computational efficiency increases significantly. Through numerical comparisons with available results, the presented method is validated. The complete three-dimensional (3D) dispersion curves are plotted in the complex wavenumber domain to gain deeper insight into the properties of evanescent waves. The dispersion characteristics of evanescent waves in various rectangular bars are illustrated. The influences of different width-to-thickness ratios on the dispersion curves are discussed in detail.

\section{MATHEMATICS AND FORMULATION OF THE PROBLEM}

In this section, we derive the analytical formulation of the problem in rectangular Cartesian coordinates $(x, y, z)$, with the origin located at a corner of the rectangular section. The $x$ axis coincides with the wave propagation direction, and the $y$ and $z$-axes are parallel to the width and thickness directions, respectively, as shown in Fig. 1. The bar of finite width $d$ and finite thickness $h$ lies in the positive $y-z$ region, where the medium occupies the region $0 \leq z \leq h$ and $0 \leq y \leq d$.

The dynamics equations (Eq. (1)) and the constitutive equa-

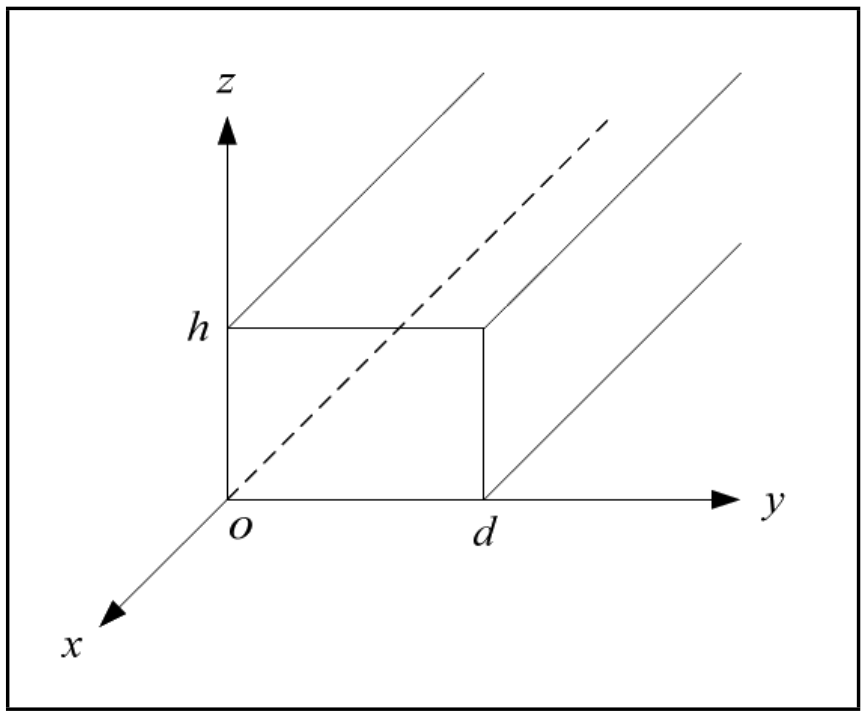

Figure 1. Schematic diagram of a bar with rectangular cross section.

tion (Eq. (2)) for an orthotropic composite bar can be expressed as follows:

$$
\begin{gathered}
\frac{\partial T_{x x}}{\partial x}+\frac{\partial T_{x y}}{\partial y}+\frac{\partial T_{x z}}{\partial z}=\rho \frac{\partial^{2} u_{x}}{\partial t^{2}} \\
\frac{\partial T_{x y}}{\partial x}+\frac{\partial T_{y y}}{\partial y}+\frac{\partial T_{y z}}{\partial z}=\rho \frac{\partial^{2} u_{y}}{\partial t^{2}} \\
\frac{\partial T_{x z}}{\partial x}+\frac{\partial T_{y z}}{\partial y}+\frac{\partial T_{z z}}{\partial z}=\rho \frac{\partial^{2} u_{z}}{\partial t^{2}}
\end{gathered}
$$

$$
\left\{\begin{array}{l}
\sigma_{x x} \\
\sigma_{y y} \\
\sigma_{z z} \\
\sigma_{y z} \\
\sigma_{x z} \\
\sigma_{x y}
\end{array}\right\}=\left[\begin{array}{cccccc}
C_{11} & C_{12} & C_{13} & 0 & 0 & 0 \\
C_{12} & C_{22} & C_{23} & 0 & 0 & 0 \\
C_{13} & C_{23} & C_{33} & 0 & 0 & 0 \\
0 & 0 & 0 & C_{44} & 0 & 0 \\
0 & 0 & 0 & 0 & C_{55} & 0 \\
0 & 0 & 0 & 0 & 0 & C_{66}
\end{array}\right]\left\{\begin{array}{c}
\varepsilon_{x x} \\
\varepsilon_{y y} \\
\varepsilon_{z z} \\
2 \varepsilon_{y z} \\
2 \varepsilon_{x z} \\
2 \varepsilon_{x y}
\end{array}\right\} .
$$

The strain-displacement relations are given as follows:

$$
\begin{aligned}
\varepsilon_{x x}=\frac{\partial u_{x}}{\partial x} ; \quad \varepsilon_{y y}=\frac{\partial u_{y}}{\partial y} ; \quad \varepsilon_{z z}=\frac{\partial u_{z}}{\partial z} \\
\varepsilon_{y z}=\frac{1}{2}\left(\frac{\partial u_{y}}{\partial z}+\frac{\partial u_{z}}{\partial y}\right) ; \quad \varepsilon_{x z}=\frac{1}{2}\left(\frac{\partial u_{x}}{\partial z}+\frac{\partial u_{z}}{\partial x}\right) \\
\varepsilon_{x y}=\frac{1}{2}\left(\frac{\partial u_{x}}{\partial y}+\frac{\partial u_{y}}{\partial x}\right) .
\end{aligned}
$$

In Eqs. (1)-(3), $\sigma_{i j}$ and $\varepsilon_{i j}$ are the stress and strain tensors, respectively, $C_{i j}$ is the elastic parameter, $\rho$ is the mass density, and $u_{i}(i=x, y, z)$ denotes the mechanical displacement component in the $i$-th direction.

For a wave propagating in the $x$-direction, we assume displacement components to be of the form,

$$
\begin{aligned}
& u_{x}(x, y, z, t)=\exp (\mathrm{i} k x-\mathrm{i} \omega t) U(y, z) \\
& u_{y}(x, y, z, t)=\exp (\mathrm{i} k x-\mathrm{i} \omega t) V(y, z) \\
& u_{z}(x, y, z, t)=\exp (\mathrm{i} k x-\mathrm{i} \omega t) W(y, z)
\end{aligned}
$$

where $U, V$, and $W$ denote the amplitude of the vibration in the $x$-, $y$-, and $z$-directions, respectively, $k$ is the wavenumber, $\omega$ is the angular frequency, and $i$ is the imaginary number. 
To meet the stress-free boundary conditions, namely $\left.\sigma_{z z}\right|_{z=0, h}=0,\left.\sigma_{x z}\right|_{z=0, h}=0,\left.\sigma_{y z}\right|_{z=0, h}=0,\left.\sigma_{y y}\right|_{y=0, d}=0$, $\left.\sigma_{y z}\right|_{y=0, d}=0$, and $\left.\sigma_{x y}\right|_{y=0, d}=0$, we introduce the positiondependent material parameters, which are given as follows:

$$
C_{i j}=C_{i j} F(y, z) ; \quad \rho=\rho F(y, z) .
$$

$F(y, z)$ is defined by

$$
F(y, z)=\pi(y) f(z)= \begin{cases}1, & 0 \leq y \leq d \text { and } 0 \leq z \leq h \\ 0, & \text { elsewhere }\end{cases}
$$

with $\pi(y)$ and $f(z)$ being rectangular window functions, $\pi(y)=\left\{\begin{array}{ll}1, & 0 \leq y \leq d \\ 0, & \text { elsewhere }\end{array}\right.$ and $f(z)= \begin{cases}1, & 0 \leq z \leq h \\ 0, & \text { elsewhere }\end{cases}$

The abovementioned boundary conditions can be automatically incorporated in the constitutive relations. ${ }^{35}$ For a simple plate structure, function $F$ is equal to $f(z)$. To reduce the number of resolving equations, we substitute Eqs. (3)-(6) into Eq. (2) and substitute the result into Eq. (1). Consequently, the following governing differential equations in terms of displacement can be obtained:

$$
\begin{aligned}
& {\left[C_{55} U_{\prime_{z z}}-k^{2} C_{11} U+C_{66} U_{\prime} y y+\mathrm{i} k\left(C_{12}+C_{66}\right) V_{\prime^{\prime}}+\right.} \\
& \left.\mathrm{i} k\left(C_{13}+C_{55}\right) W_{\prime}\right] F(y, z)+C_{55}\left(U_{\prime z}+\mathrm{i} k W\right) F(y, z)_{\prime} z+ \\
& C_{66}\left(U_{\prime} y+\mathrm{i} k V\right) F(y, z)^{\prime} y=-\rho \omega^{2} U F(y, z) \text {; } \\
& {\left[C_{44} V_{V_{z z}}-k^{2} C_{66} V+C_{22} V_{\prime y y}+\mathrm{i} k\left(C_{12}+C_{66}\right) U_{\prime y}+\right.} \\
& \left.\left(C_{23}+C_{44}\right) W_{\prime y z}\right] F(y, z)+C_{44}\left(V_{\prime_{z}}+W_{\prime y}\right) F(y, z)_{\prime} z+ \\
& \left(\mathrm{i} k C_{12} U+C_{22} V_{\prime_{y}}+C_{23} W_{\prime}\right) F(y, z)_{\prime} y=-\rho \omega^{2} V F(y, z) \text {; } \\
& {\left[C_{33} W_{\prime z z}-k^{2} C_{55} W+C_{44} W_{\prime y y}+\mathrm{i} k\left(C_{13}+C_{55}\right) U_{\prime z}+\right.} \\
& \left.\left(C_{23}+C_{44}\right) V_{\prime y z}\right] F(y, z)+C_{44}\left(V_{\prime_{z}}+W_{\prime y}\right) F(y, z)_{\prime_{y}}+ \\
& \left(\mathrm{i} k C_{13} U+C_{23} V_{\prime_{y}}+C_{33} W_{\prime_{z}}\right) F(y, z)_{\prime}=-\rho \omega^{2} W F(y, z) \text {; }
\end{aligned}
$$

where the subscript is the partial derivative with respect to $y$ or $z$.

To solve the coupled wave equations, we expand $U(y, z)$, $V(y, z)$, and $W(y, z)$ to products of two Legendre orthogonal polynomial series, as follows:

$$
\begin{aligned}
U(y, z) & =\sum_{m, n=0}^{\infty} p_{m, n}^{1} Q_{m}(z) Q_{n}(y) \\
V(y, z) & =\sum_{m, n=0}^{\infty} p_{m, n}^{2} Q_{m}(z) Q_{n}(y) ; \\
W(y, z) & =\sum_{m, n=0}^{\infty} p_{m, n}^{3} Q_{m}(z) Q_{n}(y) ;
\end{aligned}
$$

where $p_{m, n}^{i}(i=1,2,3)$ denotes the expansion coefficients, $Q_{m}(z)=\sqrt{\frac{2 m+1}{h}} P_{m}\left(\frac{2 z-h}{h}\right)$ and $Q_{n}(y)=$ $\sqrt{\frac{2 n+1}{d}} P_{n}\left(\frac{2 y-d}{d}\right)$, with $P_{m}$ and $P_{n}$ being the $m$-th and $n$-th Legendre polynomials, respectively. All the summations will be unavoidably truncated at some finite values $m=M$ and $n=N$ when higher order terms become essentially negligible.
We substitute Eq. (8) into Eq. (7), multiply both sides of the resulting equation by $Q_{j}^{*}(y) \cdot Q_{p}^{*}(z)$, integrate over $y$ from 0 to $d$ and over $z$ from 0 to $h$, taking advantage of the orthonormality of the Legendre polynomials, and rearrange the terms in the equations into a matrix form:

$$
\begin{aligned}
k^{2} & {\left[\begin{array}{lll}
A_{11}^{n, p, m, j} & A_{12}^{n, p, m, j} & A_{13}^{n, p, m, j} \\
A_{21}^{n, p, m, j} & A_{22}^{n, p, m, j} & A_{23}^{n, p, m, j} \\
A_{31}^{n, p, m, j} & A_{32}^{n, p, m, j} & A_{33}^{n, p, m, j}
\end{array}\right]\left\{\begin{array}{l}
p_{m, n}^{1} \\
p_{m, n}^{2} \\
p_{m, n}^{3}
\end{array}\right\}+} \\
k & {\left[\begin{array}{lll}
B_{11}^{n, p, m, j} & B_{12}^{n, p, m, j} & B_{13}^{n, p, m, j} \\
B_{21}^{n, p, m, j} & B_{22}^{n, p, m, j} & B_{23}^{n, p, m, j} \\
B_{31}^{n, p, m, j} & B_{32}^{n, p, m, j} & B_{33}^{n, p, m, j}
\end{array}\right]\left\{\begin{array}{l}
p_{m, n}^{1} \\
p_{m, n}^{2} \\
p_{m, n}^{3}
\end{array}\right\}+} \\
& {\left[\begin{array}{lll}
C_{11}^{n, p, m, j} & C_{12}^{n, p, m, j} & C_{13}^{n, p, m, j} \\
C_{21}^{n, p, m, j} & C_{22}^{n, p, m, j} & C_{23, p, m, j}^{n, 2} \\
C_{31}^{n, p, m, j} & C_{32}^{n, p, m, j} & C_{33}^{n, p, m, j}
\end{array}\right]\left\{\begin{array}{l}
p_{m, n}^{1} \\
p_{m, n}^{2} \\
p_{m, n}^{3}
\end{array}\right\}=} \\
& -\omega^{2}\left[\begin{array}{ccc}
M_{n, p, m, j} & 0 & 0 \\
0 & M_{n, p, m, j} & 0 \\
0 & 0 & M_{n, p, m, j}
\end{array}\right]\left\{\begin{array}{l}
p_{m, n}^{1} \\
p_{m, n}^{2} \\
p_{m, n}^{3}
\end{array}\right\} ;
\end{aligned}
$$

which can be written more compactly as

$$
k^{2} \mathbf{A} \cdot \mathbf{P}+k^{1} \mathbf{B} \cdot \mathbf{P}+\mathbf{C} \cdot \mathbf{P}=-\omega^{2} \mathbf{M} \cdot \mathbf{P}
$$

where $\mathbf{A}, \mathbf{B}, \mathbf{C}$, and $\mathbf{M}$ are matrices of order $3(M+1)^{2} \times$ $3(N+1)^{2}$, which can be obtained by Eq. (7) and are given in the Appendix, and $\mathbf{P}=\left[\begin{array}{lll}p_{m, n}^{1} & p_{m, n}^{2} & p_{m, n}^{3}\end{array}\right]^{T}$, where $p_{m, n}^{1}$, $p_{m, n}^{2}$, and $p_{m, n}^{3}$ are the three mechanical displacement components.

It should be noted that in previous works the wave differential equations are transformed into the matrix eigenvalue problem. ${ }^{32-34}$ This is useful for the propagating mode, by specifying real $k$ and then solving for $\omega$, but fails to find complex $k$ solutions because the solving involves a multivariable search. We decompose the matrix so that the $k$ dependence of the different terms becomes more apparent, and obtain Eq. (10). To obtain all three types of roots (real, imaginary and complex), we employ a mathematical technique known as the linear companion matrix method and recast Eq. (10), leading to the following generalized eigenvalue problem:

$$
\left[\begin{array}{cc}
\mathbf{0} & \mathbf{I} \\
\mathbf{A}^{-1}(\mathbf{E}-\mathbf{C}) & -\mathbf{A}^{-1} \mathbf{B}
\end{array}\right] \cdot \mathbf{R}=k \cdot \mathbf{R}
$$

where $\mathbf{I}$ is the identity matrix, $\mathbf{0}$ is the zero matrix, $\mathbf{R}=\left[\begin{array}{ll}\mathbf{P} & k \cdot \mathbf{P}\end{array}\right]^{T}$, and $\mathbf{E}=-\omega^{2} \mathbf{M}$.

This is a typical generalized eigenvalue problem and can be easily solved using an eigensolver routine that yields the complex eigenvalues $k$. The cases presented in this paper were solved with the routine eigensolver of Mathematica (version 8.0).

It is important to note that for the Legendre polynomial series, the rectangular window functions and their derivatives are introduced into the integral kernel functions in the solution process, resulting in a long computational time to perform the large number of numerical integration calculations. To improve the computational efficiency, we present the RLPAI method, using analytical integrals instead of the numerical integration of the traditional Legendre polynomial method. 


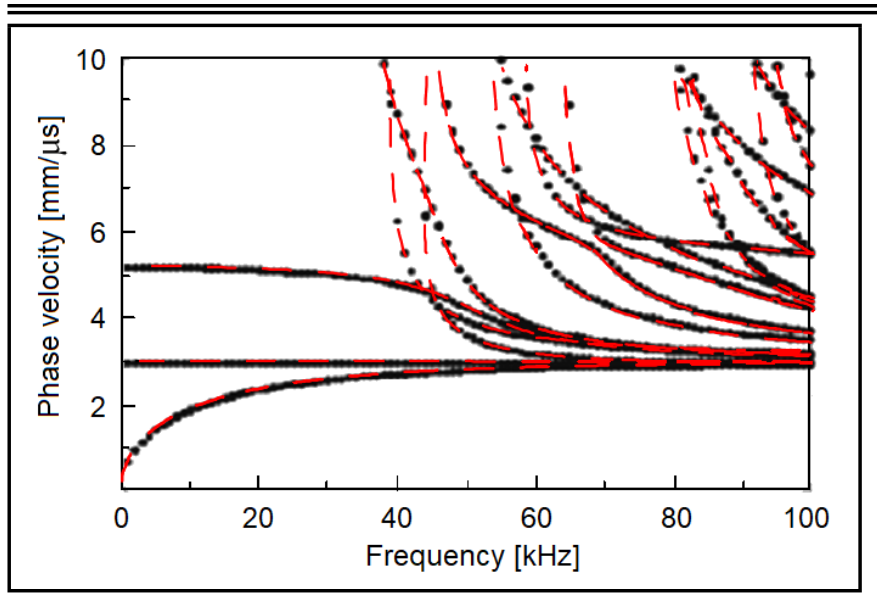

Figure 2. Phase velocity dispersion curves of a square steel bar; black dotted lines: results of Hayashi et al. obtained using the semi-analytical finite element (SAFE) method, ${ }^{37}$ red dashed lines: our results.

According to the recursion and orthogonality of the Legendre polynomial, the analytic formulas of the integrals involved in Eq. (11) can be derived. There are five integral forms:

$$
\begin{aligned}
I_{1} & =\int_{-1}^{1} t^{a} P_{n}(t) P_{m}(t) d t \\
I_{2} & =\int_{-1}^{1} t^{a} P_{n}(t) \frac{d}{d t} P_{m}(t) d t \\
I_{3} & =\int_{-1}^{1} t^{a} P_{n}(t) \frac{d^{2}}{d t^{2}} P_{m}(t) d t \\
I_{4} & =\int_{-1}^{1} t^{a} P_{n}(t) P_{m}(t) \frac{d}{d t}[h(t+1)-h(t-1)] d t \\
I_{5} & =\int_{-1}^{1} t^{a} P_{n}(t) \frac{d}{d t} P_{m}(t) \frac{d}{d t}[h(t+1)-h(t-1)] d t .
\end{aligned}
$$

All the integrals in the traditional polynomial method can be computed directly based on Eqs. (A.1)-(A.5), as shown in Appendix B. The presented method is concise, has a high computational speed, and is easy to implement. In the following section, the computational efficiency is compared between the RLPAI method and the traditional polynomial method. We take the values $M=20$ and $N=20$, if not specified.

\section{NUMERICAL RESULTS AND DISCUSSION}

\subsection{Validation of Method}

To validate the computational strategies used in this paper, we first considered wave propagation in an isotropic square steel bar. The present results are compared with the available results of Hayashi et al., from the semi-analytical finite element (SAFE) method. ${ }^{37}$ The material parameters were $C_{11}=281.757 \mathrm{GPa}, C_{12}=113.161 \mathrm{GPa}, C_{44}=84.298 \mathrm{GPa}$, $\rho=7932 \mathrm{~kg} / \mathrm{m}^{3}$, and $h=d=5.08 \mathrm{~mm}$. As shown in Fig. 2, the polynomial results match those from the SAFE method in the literature well.

The above example only considered propagating waves. Since the investigations on evanescent waves in a rectangular bar are very limited, we calculated the evanescent wave dispersion curves in an aluminum plate and compared them with the results of Giurgiutiu and Haider, which were based

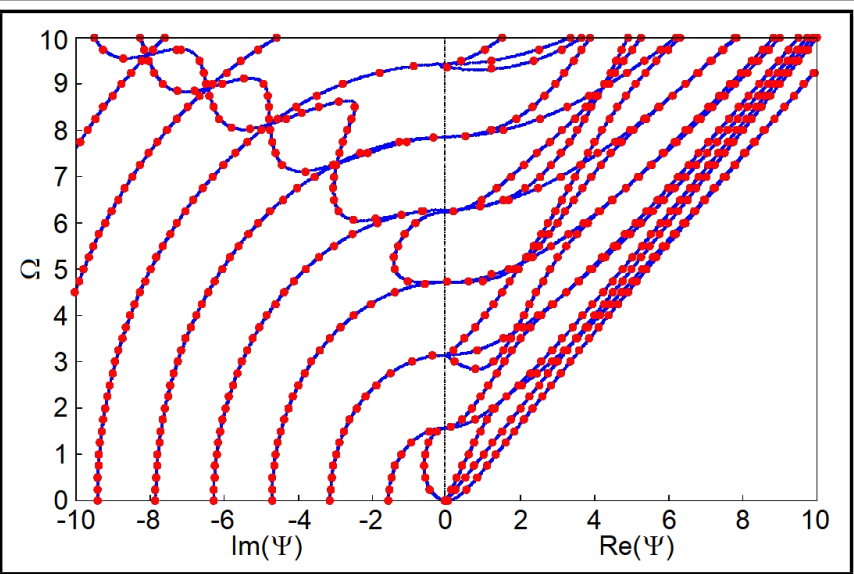

Figure 3. Dispersion curves, including real and imaginary branches, for an aluminum plate; blue lines: literature results, red dots: our results.

Table 1. Computation time of the traditional polynomial method for a bar.

\begin{tabular}{|c|c|c|c|c|c|}
\hline \multirow{2}{*}{$t$} & \multicolumn{5}{|c|}{ Truncated terms } \\
\cline { 2 - 6 } & 3 & 4 & 5 & 6 & 7 \\
\hline Integral time $t_{1}(\mathrm{~s})$ & 68.4 & 299.2 & 763.3 & 2164.8 & 15885.3 \\
Total time $t_{2}(\mathrm{~s})$ & 73.1 & 319.1 & 809.8 & 2277.1 & 16288.0 \\
Percentage $t_{1} / t_{2}$ & $93.6 \%$ & $93.8 \%$ & $94.23 \%$ & $95.1 \%$ & $97.5 \%$ \\
\hline
\end{tabular}

Table 2. Computation time of the RLPAI method for a bar.

\begin{tabular}{||c|c|c|c|c|c||}
\hline \multirow{2}{*}{$t$} & \multicolumn{5}{|c|}{ Truncated terms } \\
\cline { 2 - 6 } & 3 & 4 & 5 & 6 & 7 \\
\hline Integral time $t_{1}(\mathrm{~s})$ & 0.8 & 1.2 & 1.4 & 1.9 & 3.4 \\
Total time $t_{2}(\mathrm{~s})$ & 5.5 & 21.1 & 47.8 & 114.2 & 406.1 \\
Percentage $t_{1} / t_{2}$ & $14.5 \%$ & $5.7 \%$ & $2.9 \%$ & $1.7 \%$ & $0.8 \%$ \\
\hline
\end{tabular}

Table 3. Computation time of the traditional polynomial method for a plate.

\begin{tabular}{|c|c|c|c|c|c|}
\hline \multirow{2}{*}{$t$} & \multicolumn{5}{|c|}{ Truncated terms } \\
\cline { 2 - 6 } & 3 & 4 & 5 & 6 & 7 \\
\hline Integral time $t_{1}(\mathrm{~s})$ & 0.34 & 0.66 & 1.14 & 1.81 & 2.69 \\
Total time $t_{2}(\mathrm{~s})$ & 0.40 & 0.76 & 1.31 & 2.06 & 3.03 \\
Percentage $t_{1} / t_{2}$ & $85.0 \%$ & $86.8 \%$ & $87.0 \%$ & $87.9 \%$ & $88.8 \%$ \\
\hline
\end{tabular}

on the SAFE method. ${ }^{26}$ This served as a further verification of our method. The plate thickness was $h=1 \mathrm{~mm}$, and the non-dimensional wavenumber and frequency were $\Phi=k h / \pi$ and $\Omega=\frac{\omega h}{\pi} \sqrt{\frac{\rho}{C_{44}}}$. The material parameters were $C_{11}=103.7 \mathrm{GPa}, C_{12}=51.1 \mathrm{GPa}, C_{44}=26.3 \mathrm{GPa}$, and $\rho=2700 \mathrm{~kg} / \mathrm{m}^{3}$. The two methods are in good agreement, as shown in Fig. 3.

\subsection{Comparisons of Computational Efficiency Between Traditional Polynomial Method and Recursive Legendre Polynomial Analytical Integral (RLPAI) Method.}

In this section, we discuss the computational efficiency of the traditional polynomial method and the RLPAI method. We calculate the integral calculation time $\left(t_{1}\right)$ and the total computational time $\left(t_{2}\right)$ for various truncation terms, and the values of $t_{1}$ to $t_{2}$ for solving eigenvalues at 400 frequency points are shown in Tables 1 and 2. The numbers of terms $M$ and $N$ in Eq. (8) were the same. The CPU of the computer was an Intel Core i5-4460 with a frequency of $3.0 \mathrm{GHz}$ and $8 \mathrm{~GB}$ of memory. From Tables 1 and 2, we notice that for the tradi- 


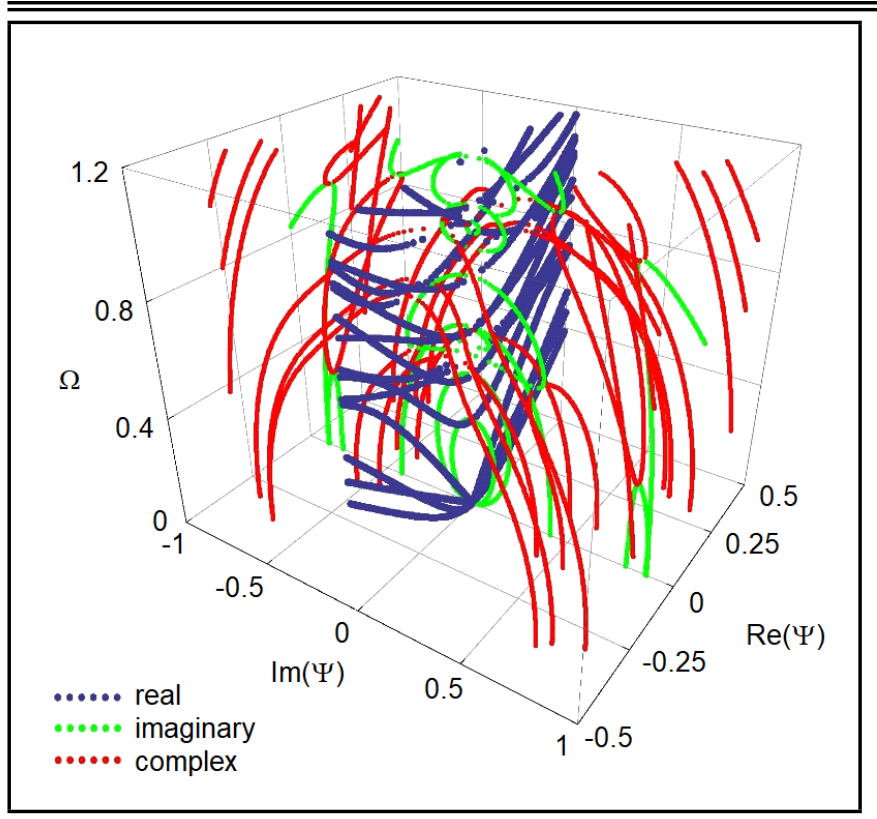

Figure 4. Three-dimensional dispersion curves for a rectangular orthotropic composite bar with $d / h=2$.

tional polynomial method, $t_{1}$ rapidly increased from 68.4 to $15885.3 \mathrm{~s}$ when the number of truncated terms varied from 3 to 7 . In contrast, $t_{1}$ and $t_{2}$ for the RLPAI method were much smaller, with values that were reduced several hundred times and even several thousand times, e.g., $15885.3 \mathrm{~s}$ compared with $3.4 \mathrm{~s}$. The proportion of $t_{1}$ to $t_{2}$ was greater than $90 \%$ for the traditional polynomial method, which means that most of the time was spent on integration calculations. The minimal proportion was less than $1 \%$ for the RLPAI method, and the time of the integral calculation was significantly reduced, which means there were significant time savings when plotting the set of dispersion curves. The comparison between the two methods indicates that the presented method can dramatically improve the computational efficiency. Moreover, for comparison, we also performed calculations for the case of a plate, as shown in Table 3. The comparison of Tables 1 and 3 shows that the integral time of the traditional polynomial method for a bar was far greater than that for a plate. A complex cross-section structure requires a greater calculation time due to the greater number of integral calculations involved.

\subsection{Complete Dispersion Curves for Rectangular Orthotropic Composite Bar}

Three-dimensional (3D) dispersion curves can provide a clearer visualization of the solutions. Figure 4 shows the complete 3D dispersion curves for a rectangular orthotropic composite bar with a width-to-thickness ratio $d / h=2$. The physical properties of the orthotropic composite material were obtained from the literature, $\rho=1580 \mathrm{~kg} / \mathrm{m}^{3}, C_{11}=C_{33}=$ $14.95 \mathrm{GPa}, C_{12}=C_{23}=6.9 \mathrm{GPa}, C_{13}=7.33 \mathrm{GPa}, C_{22}=$ $128.2 \mathrm{GPa}, C_{55}=3.81 \mathrm{GPa}$, and $C_{44}=C_{66}=6.73 \mathrm{GPa}{ }^{38}$ The purely real branches are in dark blue, purely imaginary ones are in green, and complex ones are in red. At any given frequency, there existed a finite number of propagating modes with real wavenumbers and evanescent modes with imaginary wavenumbers, as well as an infinite number of evanescent (a)

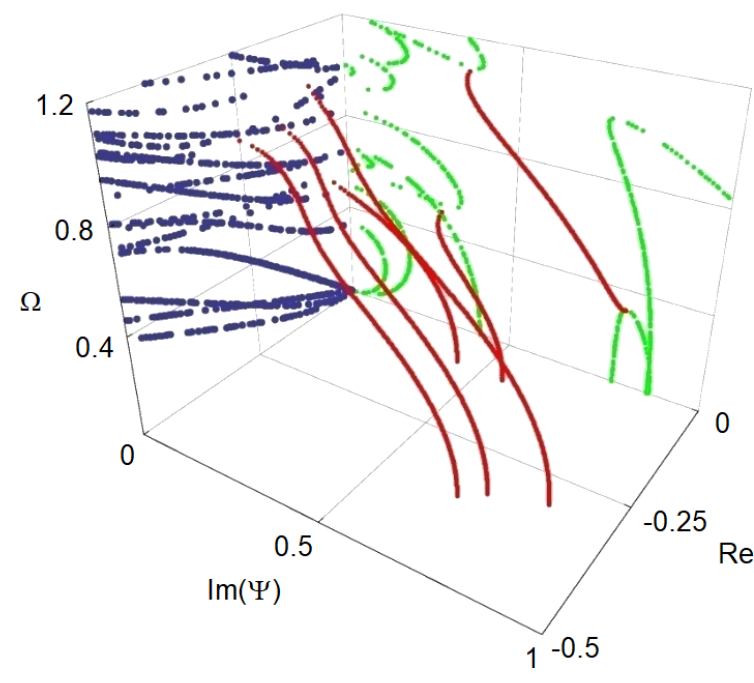

$\operatorname{Re}(\Psi)$

(b)

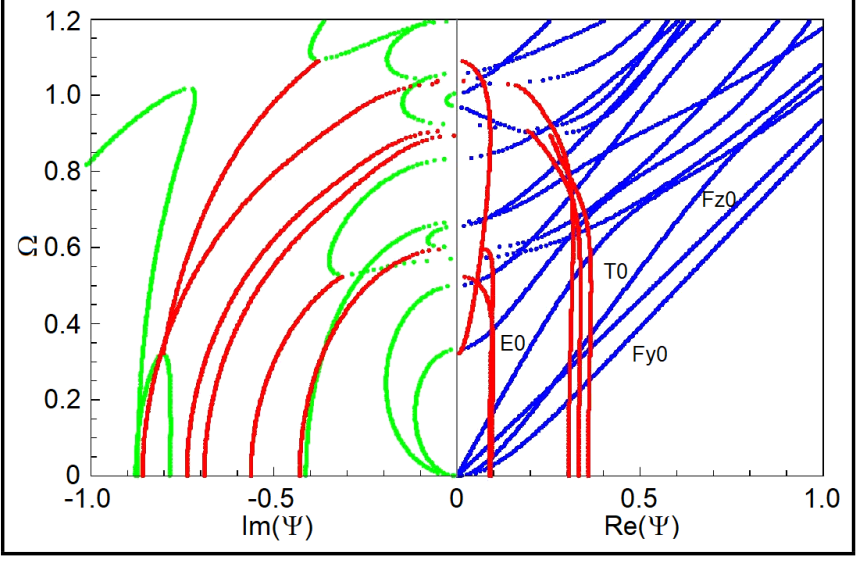

Figure 5. Dispersion curves for a rectangular orthotropic composite bar with $d / h=2$; (a) one quadrant of dispersion curves and (b) two-dimensional projection of complex branches on $\Omega-\operatorname{Re}(\Psi)$ and $\Omega-\operatorname{Im}(\Psi)$ planes.

modes with complex wavenumbers. Purely real and purely imaginary solutions occurred in pairs, while complex ones occurred in fours. To better understand the nature of these modes, we present one quadrant of the spectrum, as well as the projection of complex branches of the planes $\Omega-\operatorname{Re}(\Psi)$ and $\Omega-\operatorname{Im}(\Psi)$, as shown in Fig. 5.

Most purely imaginary modes emerged from a cutoff frequency and ended at an adjacent cut-off frequency with increasing frequency, which passed through a new cutoff frequency and transferred into real branches, namely the evanescent modes became propagating modes. A few of them with large imaginary wavenumbers emerged from a zero frequency and ended at the $\operatorname{Im}(k)$ axis or at the cutoff frequency (in the given frequency range).

There were four propagating modes below the first cutoff frequency, which were the familiar extensional modes ( $\mathrm{E}$ modes), torsional modes (T modes), flexural modes about the $y$-axis (Fy modes), and flexural modes about the $z$-axis $(\mathrm{Fz}$ modes). The first order modes of the four mode types are marked in Fig. 5(b). Most complex branches emerged from a zero frequency, and their imaginary parts progressively decreased with increasing frequency and eventually ended at the 


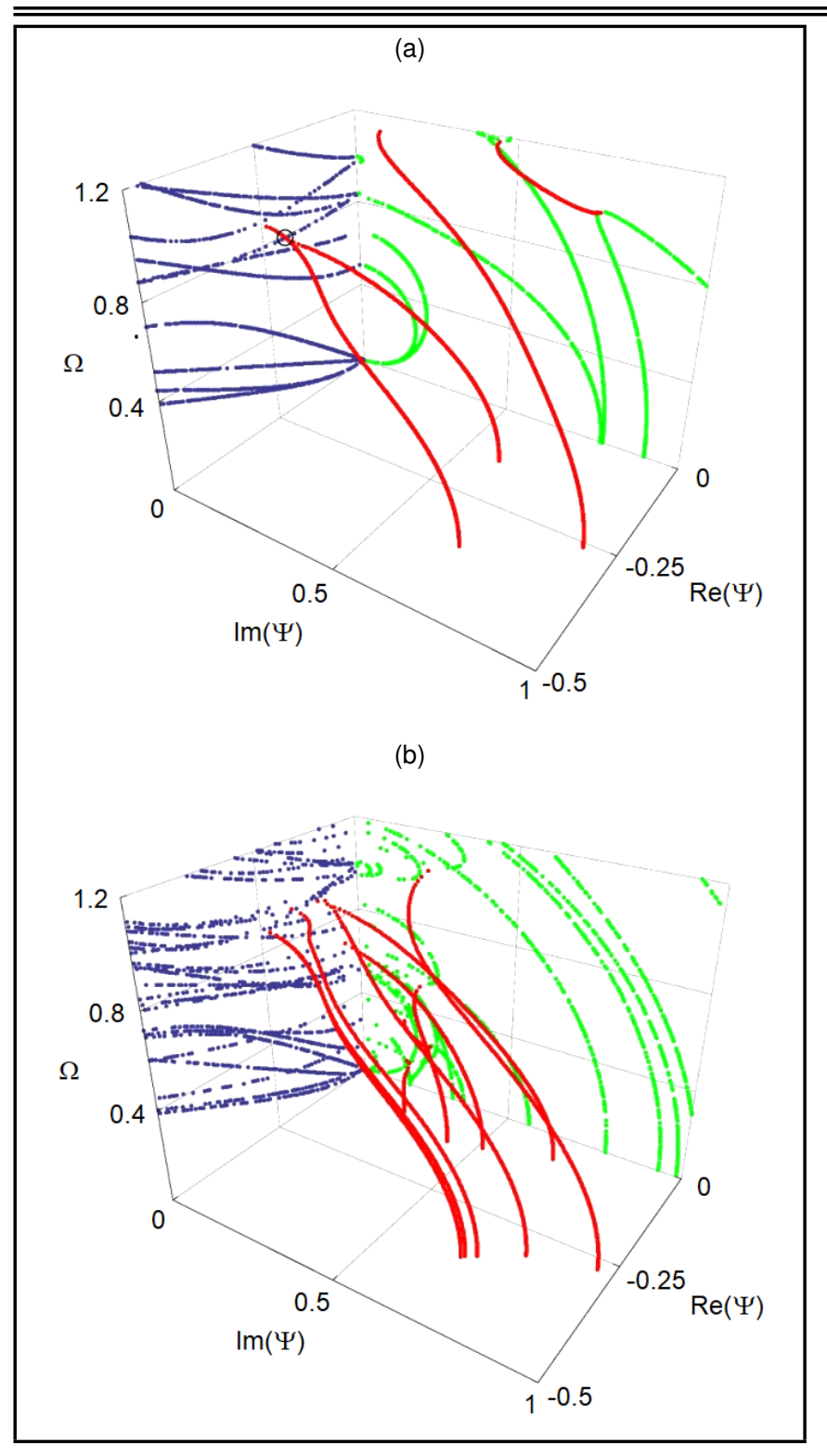

Figure 6. Dispersion curves for orthotropic composite bars: (a) $d / h=1$ and (b) $d / h=5$

minima of the real or imaginary branches. Some branches connected two purely imaginary branches, emerging from the extreme point of one imaginary branch and ending at the extreme point of the other imaginary branch, which appeared at high frequencies.

\subsection{Influence of Width-to-Thickness Ratio on Dispersion Curves}

To illustrate the influences of the width-to-thickness ratio on the dispersion curves, we performed calculations for two other rectangular orthotropic composite bars with $d / h=1$ (square bar) and $d / h=5$, as shown in Fig. 6, with the same color scheme as in Fig. 4. The comparison with Fig. 5 showed that the width-to-thickness ratio had significant influences on the guided wave dispersion, including real, imaginary, and complex branches. As the width-to-thickness ratio increased, more modes occurred (in the given frequency range), and the first local inflection point at the real branch appeared for higher-order modes. The lowest local minimum appeared on the seven real

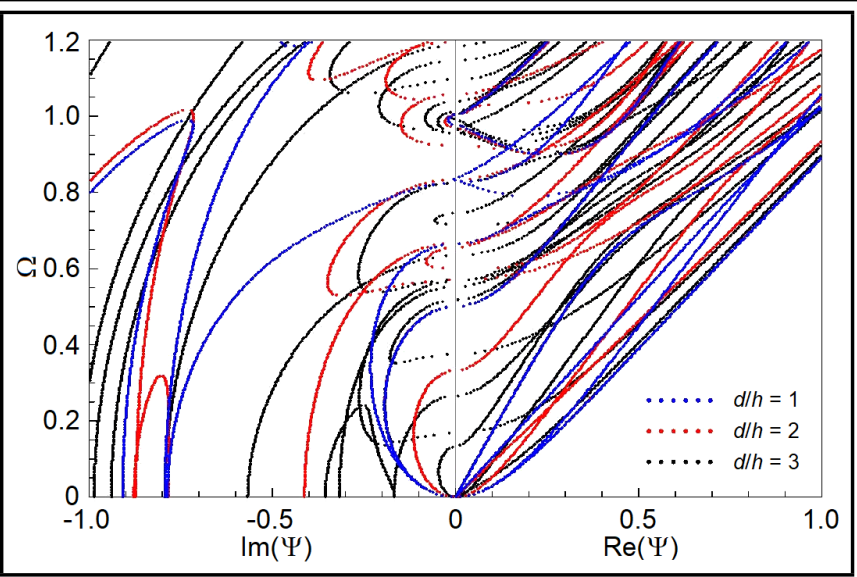

Figure 7. Dispersion curves of orthotropic composite bars with three different width to thickness ratios.

branches for a square bar, which emanated from the third cutoff frequency (marked with a circle in Fig. 6(a)).

For ease of comparison, Fig. 7 shows the dispersion curves of three orthotropic composite bars with $d / h=1, d / h=2$, andd $/ h=5$, only including real and imaginary branches. The complex branches are omitted to keep the figures clear. The plate thickness was fixed at $h=1 \mathrm{~mm}$. Evidently, the cutoff frequencies were lowered more and more as the width increased. Moreover, the lowest flexural modes were nearly identical for these three rectangular bars, which meant that such modes were not sensitive to the width. To confirm this, we also calculated the dispersion curves for an infinite plate, as shown in Fig. 8. Only purely real and imaginary branches are presented for brevity. We compare the phase dispersion curves of the first several modes for a rectangular bar with $d / h=5$ with those for an infinite flat plate, as shown in Fig. 9. The dispersion characteristics for the rectangular bar were significantly different from those for an infinite plate. For the plate, there were only three propagating modes below the first cutoff frequency, and the first cutoff frequency was larger than that of the bar. The lowest flexural mode of the rectangular bar was almost the same as that in the infinite plate. The phase velocity of the extension mode of the bar was lower than that of the plate because of the width constraint. With increasing frequency, the phase velocity of the lowest flexural (labeled as Fy0) and extensional (labeled as E0) modes tended to the Rayleigh wave velocity, like the case of the infinite plate.

\section{CONCLUSIONS}

Guided waves in rectangular orthotropic composite bars were studied using the presented RLPAI method. The positive agreement between our calculations and the available numerical results validated our method. The RLPAI method shines new light onto guided wave problems in complicated structures or complex media, which are often very demanding for conventional approaches. The conclusions obtained in this paper are summarized below.

1. An RLPAI method was successfully developed to calculate the dispersion relations of guided waves in rectangular bars, which uses analytical integration instead of 


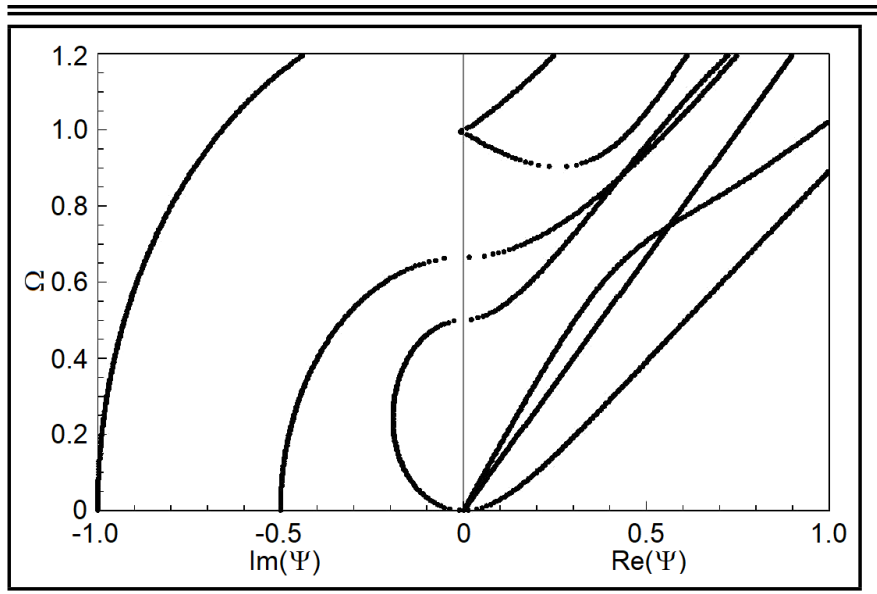

Figure 8. Dispersion curves of an infinite orthotropic composite plate.

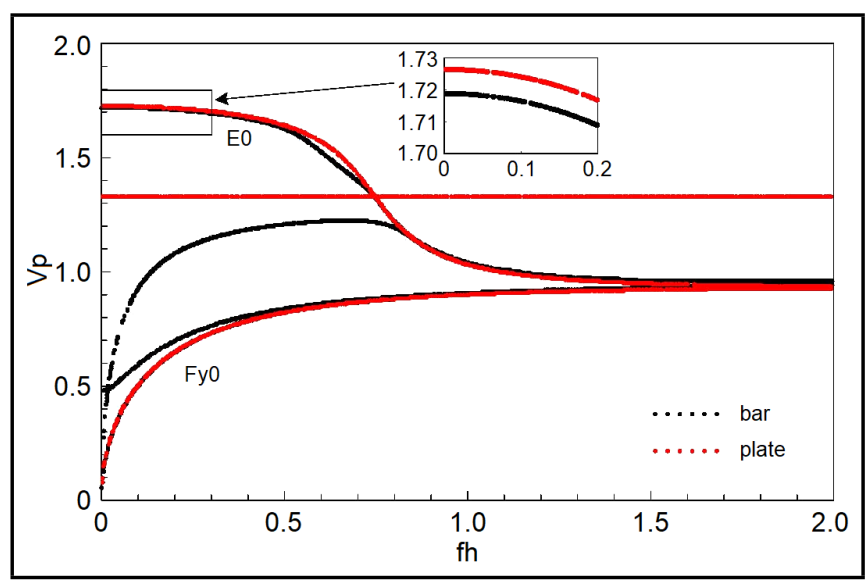

Figure 9. Phase dispersion curves of the first several modes for a bar with $d / h=5$ and an infinite plate.

numerical integration in the conventional Legendre polynomial method and dramatically improves the computational efficiency.

2. There are many purely imaginary modes starting from a cut-off frequency and ending at an adjacent cutoff frequency with increasing frequency, and some complex modes started from a zero frequency and collapsed onto the minima of the real branches. As the frequency increased, the evanescent mode became a propagating mode.

3. The width-to-thickness ratio had significant influences on guided wave dispersion, including real, imaginary, and complex branches. As the width-to-thickness ratio increased, more modes appeared and the cutoff frequency became smaller.

4. The width had almost no effect on the lowest flexural mode (flexure about the width axis). The phase velocity of the lowest extensional mode for a rectangular bar was smaller than that for an infinite plate. With increasing frequency, the phase velocity of the lowest flexural and extensional modes tended to the Rayleigh wave velocity, like the case of an infinite plate.

\section{ACKNOWLEDGMENTS}

The work was supported by the National Natural Science Foundation of China (No. 51975189, the Training Plan of Young Key Teachers of Universities in Henan Province (No. 2018-GGJS-060), and the Fundamental Research Funds for Henan Polytechnic University (No. NSFRF210408). We thank LetPub (www.letpub.com) for its linguistic assistance during the preparation of this manuscript.

\section{REFERENCES}

1 Pochhammer, L. On the velocity of propagation of small vibrations in an isotropic cylinder of infinite length, Journal Fur Die Reine Und Angewandte Mathematik, 81, 324-336, (1876). https://dx.doi.org/10.1515/9783112347287-019

2 Chree, C. Longitudinal vibrations of a circular bar, The Quarterly Journal of Pure and Applied Mathematics, 21 (83), 287-298, (1886). Retrieved from https://www.deutsche-digitale-bibliothek.de/item/ E22K4H7LIB4B2EPCOAC4ACKTWYNVKGU4 (Accessed January, 2021).

3 Rayleigh, L. On the free vibrations of an infinite plate of homogeneous isotropic elastic matter, Proceedings of the London Mathematical Society, 20 (1), 225-237, (1889). https://dx.doi.org/10.1112/plms/s1-20.1.225

${ }^{4}$ Lamb, H. On waves in an elastic plate, Proceedings of the Royal Society A, 93 (648), 114-128, (1917). https://dx.doi.org/10.1098/rspa.1917.0008

5 Peddeti, K. and Santhanam, S. Dispersion curves for Lamb wave propagation in prestressed plates using a semi-analytical finite element analysis, Journal of the Acoustical Society of America, 143 (2), 829-840, (2018). https://dx.doi.org/10.1121/1.5023335

6 Brennan, D., Arvin, E., and Salvatore, S. Computation of propagating and non-pro-pagating guided modes in nonuniformly stressed plates using spectral methods, Journal of the Acoustical Society of America, 143 (6), 3220-3230, (2018). https://dx.doi.org/10.1121/1.5040140

7 Kamal, A. and Giurgiutiu, V. Stiffness Transfer Matrix Method (STMM) for stable dispersion curves solution in anisotropic composites, Proc. The Society of Photo-Optical Instrumentation Engineers (SPIE), 9064, 906410, (2014). https://dx.doi.org/10.1117/12.2044789

8 Zhu, J., Chen, W. Q., and Ye, G. R. Reverberationray matrix analysis for wave propagation in multiferroic plates with imperfect interfacial bonding, Ultrasonics, 52 (1), 125-132, (2012). https://dx.doi.org/10.1016/j.ultras.2011.07.004

9 Cao, X., Jia, J., and Ru, Y. Asymptotic analytical solution for horizontal shear waves in a piezoelectric elliptic cylinder shell, Acta Mechanica, 226 (10), 3387-3400, (2015). https://dx.doi.org/10.1007/s00707-015-1378-3 
10 Morse, R. W. Dispersion of compressional waves in isotropic rods of rectangular cross section, Journal of the Acoustical Society of America, 20 (6), 833-838, (1948). https://dx.doi.org/10.1121/1.1906445

11 Morse, R. W. The velocity of compressional waves in rods of rectangular cross section, Journal of the Acoustical Society of America, 22 (2), 219-223, (1950). https://dx.doi.org/10.1121/1.1906592

12 Booker, R. E. Velocity dispersion of isotropic rods of square cross section vibrating in the lowest-order longitudinal mode, Journal of the Acoustical Society of America, 45 (5), 1284-1286, (1969). https://dx.doi.org/10.1121/1.1911605

13 Booker, R. E. Velocity dispersion of the lowest-order longitudinal mode in finite rods of square cross section, Journal of the Acoustical Society of America, 49 (5B), 1671-1672, (1971). https://dx.doi.org/10.1121/1.1912548

14 Nigro, N. J. Steady-state wave propagation in infinite bars of noncircular cross section, Journal of the Acoustical Society of America, 40 (6), 1501-1508, (1966). https://dx.doi.org/10.1121/1.1910255

15 Aalami, B. Waves in prismatic guides of arbitrary cross section, Journal of Applied Mechanics, 40 (4), 1067-1072, (1973). https://dx.doi.org/10.1115/1.3423127

16 Taweel, H., Dong, S. B., and Kazic, M. Wave reflection from the free end of a cylinder with an arbitrary cross-section, International Journal of Solids and Structures, 37 (2), 1701-1726, (2000). https://dx.doi.org/10.1016/S0020-7683(98)00301-1

17 Gunawan, A. and Hirose, S. Boundary element analysis of guided waves in a bar with an arbitrary cross-section, Engineering Analysis with Boundary Elements, 29 (10), 913-924, (2005). https://dx.doi.org/10.1016/j.enganabound.2005.05.007

18 Veres, I. A. and Sayir, M. B. Wave propagation in a wooden bar, Ultrasonics, 42 (1-9), 495-499, (2004). https://dx.doi.org/10.1016/j.ultras.2004.01.027

19 Hayashi, T., Suyama, M., and Abe, T. Modal analysis of guided waves in a bar with arbitrary crosssection, Ultrasonics, 72 (724), 1935-1940, (2006). https://dx.doi.org/10.1299/kikaia.72.1935

20 Manzanares-Martinez, B., Ramos-Mendieta, F., and Baltazar, A. Ultrasonic elastic modes in solid bars: An application of the plane wave expansion method, Journal of the Acoustical Society of America, 127 (6), 3503-3510, (2010). https://dx.doi.org/10.1121/1.3373402

21 Krushynska, A. A. and Meleshko, V. V. Normal waves in elastic bars of rectangular cross section, Journal of the Acoustical Society of America, 129 (3), 1324-1335, (2011). https://dx.doi.org/10.1121/1.3531800
22 Auld, B. A. Acoustic Fields and Waves in Solids, 2nd ed., Krieger Publishing Company, Malabar, Florida, USA, (1990).

23 Pagneux, V. and Maurel, A. Determination of Lamb mode eigenvalues, Journal of the Acoustical Society of America, 110 (3), 1307-1314, (2001). https://dx.doi.org/10.1121/1.1391248

24 Quintanilla, F. H., Lowe, M. J. S., and Craster, R. V. Full 3D dispersion curve solutions for guided waves in generally anisotropic media, Journal of Sound and Vibration, 363, 545-559, (2016). https://dx.doi.org/10.1016/j.jsv.2015.10.017

25 Yan, X. and Yuan, F. G. A semi-analytical approach for SH guided wave mode conversion from evanescent into propagating, Ultrasonics, 84, 430-437, (2018). https://dx.doi.org/10.1016/j.ultras.2017.12.006

26 Giurgiutiu, V. and Haider, M. F. Propagating evanescent and complex wavenumber guided waves in highperformance composites, Materials, 12 (2), 269, (2019). https://dx.doi.org/10.3390/ma12020269

27 Daros, C. H. On modelling SH-waves in a class of inhomogeneous anisotropic media via the Boundary Element Method, ZAMM-Zeitschrift fur Angewandte Mathematik und Mechanik, 90 (2), 113-121, (2010). https://dx.doi.org/10.1002/zamm.200900294

28 Mater, O. B., Gasmi, N., Zhou, H., Marc, T., and Talbi, A. Legendre and Laguerre polynomial approach for modeling of wave propagation in layered magneto-electro-elastic media, Journal of the Acoustical Society of America, 133 (3), 1415-1424, (2013). https://dx.doi.org/10.1121/1.4776198

29 Othmani, C., Dahmen, S., Njeh, A., and Ben Ghozlen, M. H. Investigation of guided waves propagation in orthotropic viscoelastic carbon-epoxy plate by Legendre polynomial method, Mechanics Research Communications, 74, 27-33, (2016). https://dx.doi.org/10.1016/j.mechrescom.2016.03.007

30 Elmaimouni, L., Lefebvre, J. E., Zhang, V., and Gryba, T. Guided waves in radially graded cylinders: A polynomial approach, NDT \& E International, 38 (5), 344-353, (2005). https://dx.doi.org/10.1016/j.ndteint.2004.10.004

31 Zhang, X. M., Li, Z., Wang, X. H., and Yu, J. G. The fractional Kelvin-Voigt model for circumferential guided waves in a viscoelastic FGM hollow cylinder, Applied Mathematical Modelling, 89, 299-313, (2021). https://dx.doi.org/10.1016/j.apm.2020.06.077

$32 \mathrm{Yu}$, J. G., Wu, B., Huo, H. L., and He, C. F. Characteristics of guided waves in anisotropic spherical curved plates, Wave Motion, 44 (4), 271-281, (2007). https://dx.doi.org/10.1016/j.wavemoti.2006.11.002

$33 \mathrm{Yu}$, J. G, Zhang, C., and Zhang, X. M. Circumferential waves in pre-stressed functionally graded 
cylindrical curved plates, Science and Engineering of Composite Materials, 21 (1), 87-97, (2014). https://dx.doi.org/10.1515/secm-2013-0042

34 Zhang, X. M., and Yu, J. G. Effects of initial stresses on guided waves in unidirectional plates, Archives of Mechanics, 65 (1), 3-26, (2013). Retrieved from https://am.ippt.pan.pl/am/article/view/v65p3 (Accessed January, 2021).

35 Lefebvre, J. E., Yu, J. G., Ratolojanahary, F. E., Xu, W. J., and Gryba, T. Mapped orthogonal functions method applied to acoustic waves-based devices, AIP Advances, 6 (6), 065307, (2016). https://dx.doi.org/10.1063/1.4953847

36 Wang, W. Introduction, The Yuanpei Program in Peking University, Peking University Press, Beijing, China, (2014). https://dx.doi.org/10.1007/978-3-642-37515-6_1

37 Hayashi, T., Song, W. J., and Rose, J. L. Guided wave dispersion curves for a bar with an arbitrary cross-section, a rod and rail example, Ultrasonics, 41 (3), 175-183, (2003). https://dx.doi.org/10.1016/S0041-624X(03)00097-0

38 Rose, J. L. Ultrasonic Waves in Solid Media, Cambridge University Press, Cambridge, UK,(1999). Retrieved from http://assets.cambridge.org/97805215/48892/frontmatter/ 9780521548892_frontmatter.pdf (Accessed January, 2021).

\section{APPENDIX A}

The explicit expressions for the element are:

$$
\begin{aligned}
A_{11}^{n, p, m, j}= & -C_{11} u(m, n, 0,0, i, j, 0,0) \\
A_{22}^{n, p, m, j}= & -C_{66} u(m, n, 0,0, i, j, 0,0) \\
A_{33}^{n, p, m, j}= & -C_{55} u(m, n, 0,0, i, j, 0,0) ; \\
A_{12}^{n, p, m, j}= & A_{21}^{n, p, m, j}=0 ; \\
A_{13}^{n, p, m, j}= & A_{31}^{n, p, m, j}=0 ; \\
A_{23}^{n, p, m, j}= & A_{32}^{n, p, m, j}=0 ; \\
B_{12}^{n, p, m, j}= & \mathrm{i}\left(C_{12}+C_{66}\right) u(m, n, 0,0, i, j, 0,1)+ \\
& \mathrm{i} C_{66} k_{y}(m, n, 0,0, i, j, 0,0) ; \\
B_{13}^{n, p, m, j}= & \mathrm{i}\left(C_{13}+C_{55}\right) u(m, n, 0,0, i, j, 1,0)+ \\
& \mathrm{i} C_{55} k_{z}(m, n, 0,0, i, j, 0,0) ; \\
B_{21}^{n, p, m, j}= & \mathrm{i}\left(C_{12}+C_{66}\right) u(m, n, 0,0, i, j, 0,1)+ \\
& \mathrm{i} C_{12} k_{y}(m, n, 0,0, i, j, 0,0) ; \\
& \mathrm{i} C_{13} k_{z}(m, n, 0,0, i, j, 0,0) ; \\
B_{31}^{n, p, m, j}= & \mathrm{i}\left(C_{13}+C_{55}\right) u(m, n, 0,0, i, j, 1,0)+ \\
B_{11}^{n, p, m, j}= & 0 ; \\
B_{22}^{n, p, m, j}= & 0 ; \\
B_{23}^{n, p, m, j}= & B_{32}^{n, p, m, j}=0 ; \\
B_{33}^{n, p, m, j}= & 0 ;
\end{aligned}
$$

$$
\begin{aligned}
C_{11}^{n, p, m, j}= & C_{55} u(m, n, 0,0, i, j, 2,0)+ \\
& C_{66} u(m, n, 0,0, i, j, 0,2)+ \\
& C_{55} k_{z}(m, n, 0,0, i, j, 1,0)+ \\
& C_{66} k_{y}(m, n, 0,0, i, j, 0,1) ; \\
C_{22}^{n, p, m, j}= & C_{44} u(m, n, 0,0, i, j, 2,0)+ \\
& C_{22} u(m, n, 0,0, i, j, 0,2)+ \\
& C_{44} k_{z}(m, n, 0,0, i, j, 1,0)+ \\
& C_{22} k_{y}(m, n, 0,0, i, j, 0,1) ; \\
C_{23}^{n, p, m, j}= & \left(C_{23}+C_{44}\right) u(m, n, 0,0, i, j, 1,1)+ \\
& C_{44} k_{z}(m, n, 0,0, i, j, 0,1)+ \\
& C_{23} k_{y}(m, n, 0,0, i, j, 1,0) ; \\
C_{32}^{n, p, m, j}= & \left(C_{23}+C_{44}\right) u(m, n, 0,0, i, j, 1,1)+ \\
& C_{23} k_{z}(m, n, 0,0, i, j, 0,1)+ \\
& C_{44} k_{y}(m, n, 0,0, i, j, 1,0) ; \\
C_{33}^{n, p, m, j}= & C_{33} u(m, n, 0,0, i, j, 2,0)+ \\
& C_{44} u(m, n, 0,0, i, j, 0,2)+ \\
& C_{33} k_{z}(m, n, 0,0, i, j, 1,0)+ \\
& C_{44} k_{y}(m, n, 0,0, i, j, 0,1) ; \\
C_{n, p, m, m}^{n, p, m, j}= & C_{21}^{n, p, m, j}=0 ; \\
31 & \rho u(m, m, j=0 ; 0,0, i, j, 0,0) ; \\
&
\end{aligned}
$$

where

$$
\begin{aligned}
& u(m, n, o, p, i, j, k, l)= \\
& \int_{0}^{d} \int_{0}^{h} Q_{i}^{*}(z) Q_{j}^{*}(y) z^{o} y^{p} \frac{\partial^{k+l} Q_{m}(z) Q_{n}(y)}{\partial z^{k} \partial y^{l}} d z d y \\
& k_{z}(m, n, o, p, i, j, k, l)= \\
& \quad \int_{0}^{d} \int_{0}^{h} Q_{i}^{*}(z) Q_{j}^{*}(y) z^{o} y^{p} \frac{\partial F(y, z)}{\partial z} \frac{\partial^{k+l} Q_{m}(z) Q_{n}(y)}{\partial z^{k} \partial y^{l}} d z d y ; \\
& k_{y}(m, n, o, p, i, j, k, l)= \\
& \int_{0}^{d} \int_{0}^{h} Q_{i}^{*}(z) Q_{j}^{*}(y) z^{o} y^{p} \frac{\partial F(y, z)}{\partial y} \frac{\partial^{k+l} Q_{m}(z) Q_{n}(y)}{\partial z^{k} \partial y^{l}} d z d y .
\end{aligned}
$$

\section{APPENDIX B}

$I_{4}$ and $I_{5}$ can be obtained based on the properties of the Heaviside function $h(t)$, yielding the following:

$$
\begin{aligned}
& I_{4}=(-1)^{a} P_{n}(-1) P_{m}(-1)-P_{n}(1) P_{m}(1) ; \\
& I_{5}=(-1)^{a} P_{n}(-1)\left[\frac{d}{d t} P_{m}(t)\right]_{t=-1}-P_{n}(1)\left[\frac{d}{d t} P_{m}(t)\right]_{t=1} ;
\end{aligned}
$$

where

$$
\left[\frac{d}{d t} P_{n}(t)\right]_{t=-1}=(-1)^{n+1} \frac{\Gamma(n+2)}{2 \Gamma(n)} .
$$

Rewriting $P_{m}(t)$ and invoking orthogonality, ${ }^{36}$ we obtain

$$
P_{m}(t)=\sum_{s=0}^{m / 2}(-1)^{s} \frac{(2 m-2 s) !}{2^{m} s !(m-s) !(m-2 s) !} t^{m-2 s} .
$$


When $a+m \geq n, a+m-n=$ even, and $m-2 s+a=n+2 p, \quad$ and

$$
\begin{aligned}
I_{1}= & \int_{-1}^{1} t^{a} P_{n}(t) P_{m}(t) d t= \\
& \sum_{s=0}^{m / 2}(-1)^{s} \frac{(2 m-2 s) !}{2^{m} s !(m-s) !(m-2 s) !} \frac{2^{n+1}(n+2 p) !(n+p) !}{p !(2 n+2 p+1) !} .
\end{aligned}
$$

Otherwise, $I_{1}=0$.

When $a+m-1 \geq n, a+m-n-1=$ even, and $m-$ $2 s+a-1=n+2 p$,

$$
\begin{aligned}
I_{2}= & \int_{-1}^{1} t^{a} P_{n}(t) \frac{d}{d t} P_{m}(t) d t= \\
& \sum_{s=0}^{m / 2}(-1)^{s} \frac{(2 m-2 s) !}{2^{m} s !(m-s) !(m-2 s) !} \frac{2^{n+1}(n+2 p) !(n+p) !}{p !(2 n+2 p) !} .
\end{aligned}
$$

Otherwise, $I_{2}=0$.

When $a+m-2 \geq n, a+m-n-2=$ even, and $m-$ $2 s+a-2=n+2 p$,

$$
\begin{aligned}
I_{3}= & \int_{-1}^{1} t^{a} P_{n}(t) \frac{d^{2}}{d t^{2}} P_{m}(t) d t= \\
& \sum_{s=0}^{m / 2}(-1)^{s} \frac{(2 m-2 s) !}{2^{m} s !(m-s) !(m-2 s) !} \frac{2^{n+1}(n+2 p) !(n+p) !}{p !(2 n+2 p-1) !} .
\end{aligned}
$$

Otherwise $I_{3}=0$.

Actually,

$$
\begin{aligned}
& u(m, n, o, p, i, j, k, l)= \\
& \int_{0}^{d} \int_{0}^{h} Q_{i}^{*}(z) Q_{j}^{*}(y) z^{o} y^{p} \frac{\partial^{k+l} Q_{m}(z) Q_{n}(y)}{\partial z^{k} \partial y^{l}} d z d y= \\
& \int_{0}^{h} Q_{i}^{*}(z) z^{o} \frac{\partial^{k} Q_{m}(z)}{\partial z^{k}} d z \times \int_{0}^{d} Q_{j}^{*}(y) y^{p} \frac{\partial^{l} Q_{n}(y)}{\partial y^{l}} d y ; \\
& k_{z}(m, n, o, p, i, j, k, l)= \\
& \int_{0}^{d} \int_{0}^{h} Q_{i}^{*}(z) Q_{j}^{*}(y) z^{o} y^{p} \frac{\partial F(y, z)}{\partial z} \frac{\partial^{k+l} Q_{m}(z) Q_{n}(y)}{\partial z^{k} \partial y^{l}} d z d y= \\
& \int_{0}^{h} Q_{i}^{*}(z) z^{o} \frac{\partial^{k} Q_{m}(z)}{\partial z^{k}} \frac{\partial f(z)}{\partial z} d z \times \int_{0}^{d} Q_{j}^{*}(y) y^{p} \frac{\partial^{l} Q_{n}(y)}{\partial y^{l}} d y
\end{aligned}
$$

$k_{y}(m, n, o, p, i, j, k, l)=$

$\int_{0}^{d} \int_{0}^{h} Q_{i}^{*}(z) Q_{j}^{*}(y) z^{o} y^{p} \frac{\partial F(y, z)}{\partial y} \frac{\partial^{k+l} Q_{m}(z) Q_{n}(y)}{\partial z^{k} \partial y^{l}} d z d y=$ $\int_{0}^{h} Q_{i}^{*}(z) z^{o} \frac{\partial^{k} Q_{m}(z)}{\partial z^{k}} d z \times \int_{0}^{d} Q_{j}^{*}(y) y^{p} \frac{\partial^{l} Q_{n}(y)}{\partial y^{l}} \frac{\partial \pi(y)}{\partial y} d y$.

All the integrals can be converted into two integrals multiplied by each other. Thus, the original double integral can be calculated by the above analytical integral, for example:

$u(m, n, 1,0, i, j, 0,1)=$

$\int_{0}^{d} \int_{0}^{h} Q_{i}^{*}(z) Q_{j}^{*}(y) z Q_{m}(z) \frac{\partial Q_{n}(y)}{\partial y} d z d y=$ $\int_{0}^{h} Q_{i}^{*}(z) Q_{m}(z) d z \times \int_{0}^{d} Q_{j}^{*}(y) \frac{\partial Q_{n}(y)}{\partial y} d y=$ $\int_{0}^{h} \sqrt{\frac{2 i+1}{h}} P_{i}\left(\frac{2 z-h}{h}\right) \sqrt{\frac{2 m+1}{h}} P_{m}\left(\frac{2 z-h}{h}\right) z d z \times$ $\int_{0}^{d} \sqrt{\frac{(2 i+1)(2 m+1)(2 j+1)(2 n+1)}{h^{2} d^{2}}}$. $\int_{0}^{h} P_{i}\left(\frac{2 z-h}{h}\right) P_{m}\left(\frac{2 z-h}{h}\right) z d z \times$ $\int_{0}^{d} P_{j}\left(\frac{2 y-d}{d}\right) \frac{\partial P_{n}\left(\frac{2 y-d}{d}\right)}{\partial y} d y=$ $\frac{1}{2 d} \sqrt{(2 i+1)(2 m+1)(2 j+1)(2 n+1)}$. $\int_{-1}^{1} P_{i}(t) P_{m}(t)(t+1) d t \times \int_{-1}^{1} P_{j}(s) \frac{\partial P_{n}(s)}{\partial s} d s=$ $\frac{1}{2 d} \sqrt{(2 i+1)(2 m+1)(2 j+1)(2 n+1)}$.

$$
\begin{gathered}
\left(\int_{-1}^{1} t P_{i}(t) P_{m}(t) d t+\int_{-1}^{1} P_{i}(t) P_{m}(t) d t\right) \times \\
\int_{-1}^{1} P_{j}(s) \frac{\partial P_{n}(s)}{\partial s} d s .
\end{gathered}
$$

